\title{
FATORES ASSOCIADOS À MULTIMORBIDADE DE IDOSOS INTERNADOS EM UM HOSPITAL DE ENSINO DO PARANÁ
}

Pamela Tainá Licoviski; Hospital Universitário Regional dos Campos Gerais - Universidade Estadual de Ponta Grossa (UEPG); pamelalicoviski@gmail.com;

Dreli Gonçalves'; Universidade Estadual de Ponta Grossa (UEPG); driquinha_pg@hotmail.com; Cloris Regina Blanski Grden²; Universidade Estadual de Ponta Grossa (UEPG); reginablanski@hotmail.com; Luciane Patrícia Andreani Cabral33; Universidade Estadual de Ponta Grossa (UEPG); Luciane.pacabral@gmail.com; Melina Lopes Lima4; Universidade Estadual de Ponta Grossa (UEPG); enfmelina@gmail.com; Danielle Bordin ${ }^{5}$; Universidade Estadual de Ponta Grossa (UEPG); daniellebordin@hotmail.com.

\section{RESUMO}

Introdução: $\mathrm{O}$ envelhecimento ocasiona diversas alterações ao corpo humano, gerando vulnerabilidade, como diminuição da capacidade funcional e incidência de doenças, como as crônicas não transmissíveis. Objetivo: Analisar prevalência e os fatores associados à multimorbidade de idosos internados em um hospital de ensino, segundo características sociodemográficas, estilo de vida e utilização de serviços na rede de atenção à saúde. Métodos: É um estudo transversal, qualitativo, analítico. A amostra foi composta por 144 idosos, que foram internados em um hospital universitário paranaense. Os dados foram coletados 30 dias pós alta hospitalar, via telefone. A variável dependente foi ocorrência de multimorbidade e as independentes características sociodemográficas, estilo de vida e utilização de serviços de saúde hospitalares. Realizou-se análise de regressão logística. Resultados: Verificou-se que 55\% dos idosos investigados apresentaram multimorbidade. Apresentaram maiores chances de dispor de multimorbidades idosos com baixa escolaridade $(\mathrm{OR}=16,29)$, de cor não branca $(\mathrm{OR}=2,34)$, que ficaram internados por um período de 4 a 7 dias $(\mathrm{OR}=6,91)$ e mais de 7 dias $(\mathrm{OR}=3,03)$, que necessitaram de consulta de retorno pós alta hospitalar $(\mathrm{OR}=18,99)$ e que, no domicílio, precisaram de ajuda de alguém para seguir as recomendações médicas $(\mathrm{OR}=3,16)$. Conclusão: Identificou alta prevalência de multimorbidade e importantes fatores associados a multimorbidades em idosos internados, com destaque para escolaridade; cor; tempo de internação; agendamento de retorno ao hospital no pós alta e necessidade de ajuda de alguém (familiar/cuidador), no pós alta. É fundamental a atenção com idosos internados visando sua independência funcional para que possam prevenir o aparecimento de novos agravos em saúde.

Palavras-chave: Morbidade; Internação Hospitalar; Fatores de Risco; Idosos; Doenças Não Transmissíveis. 